\title{
Inter-observer agreement of computed tomography and magnetic resonance imaging on gross tumor volume delineation of intrahepatic cholangiocarcinoma: an initial study
}

\author{
Nan Zhou ${ }^{1 \#}$, Anning Hu ${ }^{1 \#}$, Zhihao Shi ${ }^{1 \#}$, Xiaolu Wang ${ }^{2}$, Qiongjie Zhu', Qun Zhou ${ }^{1}$, Jun $\mathrm{Ma}^{3}$, Feng Zhao ${ }^{4}$, \\ Weiwei Kong ${ }^{2,5}$, Jian $\mathrm{He}^{1}$ \\ ${ }^{1}$ Department of Radiology, Nanjing Drum Tower Hospital, The Affiliated Hospital of Nanjing University Medical School, Nanjing, China; ${ }^{2}$ The \\ Comprehensive Cancer Centre of Drum Tower Hospital, Medical School of Nanjing University \& Clinical Cancer Institute of Nanjing University, \\ Nanjing, China; ${ }^{3}$ Department of Mathematics, Nanjing University of Science and Technology, Nanjing, China; ${ }^{4}$ Department of Radiation Oncology, \\ the First Affiliated Hospital, Zhejiang University School of Medicine, Hangzhou, China; ${ }^{5}$ Department of Oncology of Yizheng Hospital, Nanjing \\ Drum Tower Hospital Group, Yizheng, China \\ "These authors contributed equally to this work.
}

Correspondence to: Jian He, PhD. Department of Radiology, Nanjing Drum Tower Hospital, The Affiliated Hospital of Nanjing University Medical School, No. 321 Zhongshan Road, Nanjing 210008, China. Email: hjxueren@126.com; Weiwei Kong, MD. The Comprehensive Cancer Centre of Drum Tower Hospital, Medical School of Nanjing University \& Clinical Cancer Institute of Nanjing University, Nanjing 210008, China; Department of Oncology of Yizheng Hospital, Nanjing Drum Tower Hospital Group, Yizheng 211400, China. Email: weiweikong.md@hotmail.com.

Background: Intrahepatic cholangiocarcinoma (ICC) is the second most common primary liver tumor, and local radiotherapy has a positive effect on patients with an unresectable tumor. Accurate delineation of gross tumor volume (GTV) is crucial to improve the efficacy of radiotherapy. The purpose of this article was to evaluate the consistency of CT, diffusion weighted imaging (DWI) and Gadoxetic acid disodium (GdEOB-DTPA)-enhanced MRI on GTV delineation of ICC.

Methods: Fourteen patients with ICC underwent CT (Plain and Portal, CT scans before and $70 \mathrm{~s}$ after the injection of Omnipaque, respectively), DWI, and Gd-EOB-DTPA-enhanced MRI (EOB $70 \mathrm{~s}$ and EOB $15 \mathrm{~min}, \mathrm{mDIXON}$ scans at $70 \mathrm{~s}$ and $15 \mathrm{~min}$ after the injection of Gd-EOB-DTPA, respectively) examinations before radiotherapy. Volumes of GTV delineation on CT and MRI images were recorded. Dice similarity coefficient (DSC) was calculated to evaluate the spatial overlap.

Results: Tumor volume on DWI and EOB 15 min were larger than that on EOB $70 \mathrm{~s}$ significantly (both $\mathrm{P}=0.004$ ). DSC of DWI was significantly larger than that of other CT and MRI sequences (all $\mathrm{P} \leq 0.002$ ). DSC of EOB 15 min tended to be larger than that of other CT sequences and EOB $70 \mathrm{~s}$, however, without significances (all $\mathrm{P}>0.005)$. Significant correlation was found between DSC and tumor volume $(\mathrm{R}=0.35$, $\mathrm{P}=0.003)$.

Conclusions: DWI had significantly higher agreement on GTV delineation of ICC. GTV delineations of ICC on Gd-EOB-DTPA-enhanced MRI showed excellent inter-observer agreement. Fusion of CT and MRI images should be considered to improve the accuracy of GTV delineation.

Keywords: Consistency; computed tomography (CT); magnetic resonance imaging (MRI); intrahepatic cholangiocarcinoma (ICC); gross tumor volume delineation (GTV delineation)

Submitted Dec 26, 2019. Accepted for publication Sep 28, 2020.

doi: 10.21037/qims-19-1093

View this article at: http://dx.doi.org/10.21037/qims-19-1093 


\section{Introduction}

Intrahepatic cholangiocarcinoma (ICC) is the second most common primary liver tumor with an increasing prevalence (1). The reason for the increased prevalence might be that some adenocarcinomas with unknown primary are now reclassified as ICC (1). Surgical resection is a potential curative treatment, with a 5 -year survival rate of $15 \%$ to $40 \%(1,2)$. However, lesions of ICC in many patients are beyond resectable criteria. In addition, some elderly patients cannot undergo curative surgical resection due to comorbidities (3). For those patients, local radiotherapy has a positive effect on reducing tumor burden and improving prognosis $(4,5)$.

Accurate delineation of gross tumor volume (GTV) is crucial to improve the efficacy of radiotherapy and reduce radiation-induced complications $(6,7)$. Computed tomography (CT) remains the gold standard for GTV delineation, but it has some limitations, such as insufficient contrast between tumor and adjacent soft tissue (6). Therefore, it is essential to combine techniques with high soft tissue contrast to improve the accuracy of GTV delineation.

Magnetic resonance imaging (MRI) is an advanced tool with high resolution of soft tissue, and has become the standard imaging method for the detection and characterization of liver tumors (8). Diffusion weighted imaging (DWI) reflects the thermally random motion of water molecule, which is influenced by the obstruction of cell membranes and macromolecules in tissues (9). In recent years, DWI has been widely used for the detection, diagnosis, grading, and therapy response assessment of liver tumors (10-13). Pathologically, collagen deposition and fibrosis are the characteristics of ICC (14). Fibrosis in tumor tissues limits the random motion of water molecules and improves tumor definition on DWI images. Gadoxetic acid disodium (Gd-EOB-DTPA) is a liver-specific MRI contrast agent and has hepatocyte-selective properties. Therefore, Gd-EOB-DTPA-enhanced MRI can provide both vascular phase images and hepatocyte-selective phase images, which has been applied to evaluate several liver tumors $(15,16)$. ICC originates from the intrahepatic biliary epithelium (14), which does not demonstrate uptake of GdEOB-DTPA. Therefore, there is theoretically no notable Gd-EOB-DTPA uptake by ICC in hepatocyte-selective phase, but the adjacent normal liver tissues would take in Gd-EOB-DTPA (8). This facilitates the delineation of ICC. Radiation therapy planning based on Gd-EOBDTPA-enhanced MRI has been used to preserve functional liver tissues adjacent to hepatocellular carcinoma during radiotherapy (17). Therefore, we believe that DWI and GdEOB-DTPA-enhanced MRI can contribute to the GTV delineation of ICC.

The purpose of this study was to detect the interobserver agreement of GTV delineation on CT, DWI and Gd-EOB-DTPA-enhanced MRI in ICC. Values of DWI and Gd-EOB-DTPA-enhanced MRI in GTV delineation of ICC was evaluated.

\section{Methods}

\section{Patients}

The ethical committee of Nanjing Drum Tower Hospital approved this prospective study, and all patients gave written informed consents. Fourteen patients $(7$ males and 7 females) affected by ICC were recruited between March 2017 and February 2019. Multiple liver lesions were found in three patients, and single liver lesion was found in the other eleven patients. The inclusion criteria were: (I) age $\geq 18$ years old; (II) pathologically confirmed ICC (needle biopsy, abdominal laparotomy, or postoperative pathology); (III) scheduled to undergo radiotherapy and receive CT and MRI follow-ups in our hospital. The exclusion criteria were: (I) patients with hilar cholangiocarcinoma or distal cholangiocarcinoma; (II) the recurrent lesions that had unclear boundary with the postoperative changes; (III) GdEOB-DTPA-enhanced or iodinated contrast-enhanced contraindications (e.g., GFR $<30 \mathrm{~mL} / \mathrm{min}$ or combined with severe cardiovascular disease); (IV) MRI contraindications (e.g., artificial cochlea or cardiac pacemaker implantation).

\section{CT and MRI examinations}

CT and MRI examinations for all patients were performed before radiotherapy, and the interval between CT and MRI scans was less than 2 weeks. All patients fasted for 4-8 hours before examinations. CT images were obtained using a 64-multi-detector CT scanner (LightSpeed VCT, GE Healthcare, Waukesha, USA), with supine position and head-first. The time points for the arterial, portal, and delayed CT scans were 35,70 and $180 \mathrm{~s}$ after the injection of Omnipaque $(350 \mathrm{mgI} / \mathrm{mL}$, GE Healthcare, Shanghai, China), respectively. $80-100 \mathrm{~mL}$ omnipaque was intravenously injected at a rate of $3.0 \mathrm{~mL} / \mathrm{s}$ followed by $50 \mathrm{~mL}$ saline flush using a power injector (Medrad Stellant CT Injector System; One Medrad Drive Indianola, PA, 

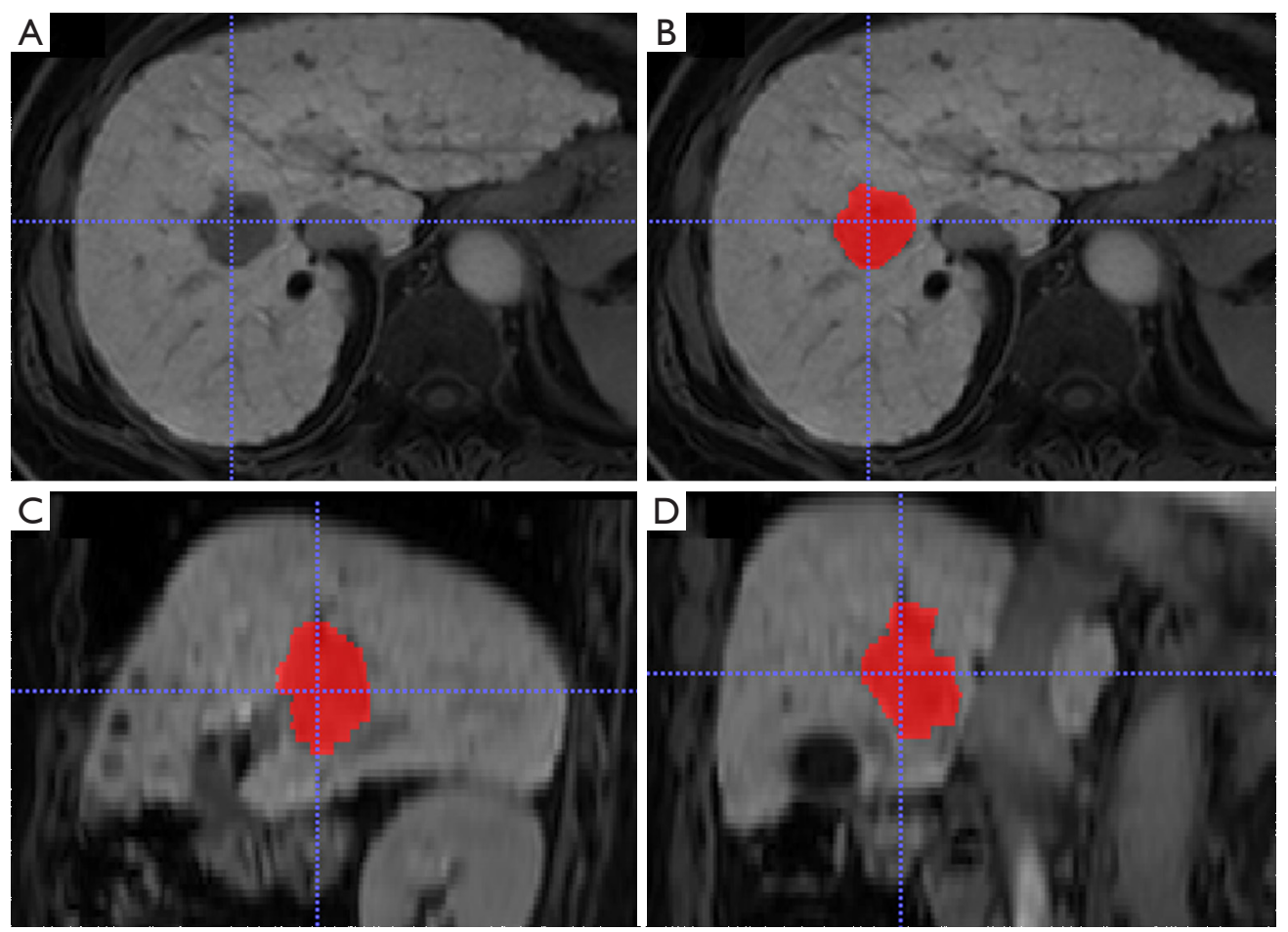

Figure 1 GTV delineation for ICC. (A) The location of ICC; (B) the delineation of GTV slice by slice; (C,D) exhibited the GTV delineation on sagittal and coronal views, respectively.

USA). Plain and Portal were defined as CT scans before and $70 \mathrm{~s}$ after the injection of Omnipaque, respectively. The slice thickness for CT images was $5 \mathrm{~mm}$.

MRI images were acquired using a 3.0-T MRI scanner (Ingenia, Philips Medical Systems, Best, the Netherlands) with a 16-channel phased-array body coil in supine position and head-first. mDIXON sequence (water, fat, in-phase, and out-phase images) with $2 \mathrm{~mm}$ slice thickness was used to obtain T1-weighted images. Gd-EOB-DTPA (Primovist; Bayer HealthCare, Berlin, Germany) was used for enhanced MRI protocol. Three-arterial-phase images (early arterial phase, middle arterial phase, and late arterial phase) were acquired at $20 \mathrm{~s}$ after the intravenous administration of GdEOB-DTPA $(0.025 \mathrm{mmol} / \mathrm{kg})$, and each arterial phase was $6 \mathrm{~s}$. Gd-EOB-DTPA was injected at a rate of $1.0 \mathrm{~mL} / \mathrm{s}$, followed by $15 \mathrm{~mL}$ saline flush with a same speed using a power injector (Medrad Spectris Solaris EP MR Injector System; One Medrad Drive Indianola, PA, USA). Portal phase images (EOB 70 s), delayed phase images, and hepatobiliary phase images (EOB $15 \mathrm{~min}$ ) were acquired using mDIXON sequence at $70 \mathrm{~s}, 180 \mathrm{~s}, 15 \mathrm{~min}$ after the injection of Gd-EOB-DTPA, respectively. A single shot echo-planar imaging (SS-EPI) DWI with b values of 0,800 , and $1,000 \mathrm{~s} / \mathrm{mm}^{2}$ was acquired, and the slice thickness was $6 \mathrm{~mm}$. DWI image with b value of $1,000 \mathrm{~s} / \mathrm{mm}^{2}$ was used for GTV delineation.

\section{Image analysis}

All CT and MRI images were transferred to ITK-SNAP software (version 3.4.0, www.itksnap.org) for analysis. GTV of ICC was independently delineated on CT and MRI images by one radiation oncologist (with 4 years of clinical experience in liver tumor radiotherapy) and three radiologists (with 3, 5 and 8 years of experience in liver imaging, respectively). GTV delineation included the visible tumor area as shown in Figure 1. For multiple hepatic lesions, only the largest one was chosen for GTV delineation. None of the four observers knew any clinical information of patients. Tumor volume was automatically calculated after GTV delineation. The average tumor volume delineated by the four observers was set as the final tumor volume. Delineation of GTV by the four observers via consensus was considered as the benchmark (18). Dice 
Table 1 Volumes and DSCs of GTV on different CT and MRI sequences

\begin{tabular}{lcl}
\hline Parameters & Mean (standard deviation) & Median (range) \\
\hline Volume $\left(\mathrm{cm}^{3}\right)$ & $92.1(112.0)$ & $69.5(3.2-442.8)$ \\
Plain & $79.3(82.5)$ & $64.4(1.8-312.8)$ \\
Portal & $117.8(120.1)$ & $97.1(1.4-441.1)$ \\
DWI & $80.5(77.7)$ & $66.9(0.6-279.1)$ \\
EOB 70 s & $105.6(117.2)$ & $84.7(3.2-443.1)$ \\
EOB 15 min & & \\
DSC & $0.78(0.24)$ & $0.89(0.00-0.97)$ \\
Plain & $0.82(0.19)$ & $0.88(0.00-0.97)$ \\
Portal & $0.90(0.09)$ & $0.92(0.51-0.97)$ \\
DWI & $0.84(0.14)$ & $0.89(0.37-0.96)$ \\
EOB 70 s & $0.85(0.11)$ & $0.89(0.37-0.96)$ \\
EOB 15 min &
\end{tabular}

DSC, dice similarity coefficient; GTV, gross tumor volume; Plain, CT scan before the injection of Omnipaque; Portal, CT scan at $70 \mathrm{~s}$ after the injection of Omnipaque; EOB $70 \mathrm{~s}, \mathrm{mDIXON}$ scan at $70 \mathrm{~s}$ after the injection of Gd-EOB-DTPA; EOB $15 \mathrm{~min}$, mDIXON scan at 15 min after the injection of Gd-EOB-DTPA.

similarity coefficient (DSC) is the measurement for the spatial overlap between two delineations A and B (19). DSC was calculated using the following equation with an inhouse program based on Matlab software (MathWorks Inc., Natick, MA, USA):

$$
D S C=\frac{2|A \cap B|}{|A|+|B|}
$$

where A was the volume of GTV for an observer and B was the volume of GTV for the benchmark. DSC $\geq 0.8$ indicated almost perfect agreement (20).

\section{Statistical analysis}

SPSS18.0 software (SPSS Inc., Chicago, IL, USA) was used for all statistical analyses. Continuous data was showed as median and range. Nonparametric paired-sample Wilcoxon signed-rank test was used to test the differences of tumor volumes and DSC among each sequence, and $\mathrm{P}<0.005$ was considered to be statistically significant (Bonferroni corrected). Spearman rank correlation test was used to evaluate the relationship between DSC and tumor volume delineated by each observer, and $\mathrm{P}<0.05$ was considered a significant difference.

\section{Results}

Tumor volumes and DSCs of each CT or MRI sequence were shown in Table 1, and the differences of tumor volumes and DSCs are shown in Table 2.

Tumor volumes on DWI and EOB 15 min were significantly larger than that on EOB $70 \mathrm{~s}$. But tumor volume delineated on other CT or MRI sequences had no significant differences.

DSC of DWI was significantly larger than that of other CT and MRI sequences. DSC of EOB 15 min tended to be larger than that of Plain, Portal and EOB 70 s, however, without significant differences.

In addition, significant correlation was found between DSC and tumor volume on all CT and MRI sequences ( $\mathrm{R}=0.35, \mathrm{P}=0.003)$.

\section{Discussion}

Tumor volume on DWI was the largest and significantly larger than that on EOB 70 s. ICC always showed a nonencapsulated mass with irregular contour and ill-defined margin $(21,22)$. In addition, some daughter nodules could be found around the primary tumor (23). Therefore, it was difficult to clearly delineate the margin of the tumor, and some peripheral tumor tissues were excluded from GTV delineation. Pathologically, ICC always shows peripheral hypercellularity and central hypocellularity with fibrous component (24). This causes peripheral hyperintensity and central hypointensity on DWI images with high $b$-value (25). Therefore, we considered that the margin of ICC on DWI could display more clearly than other CT and MRI sequences, and more peripheral tumor tissues could be included on GTV delineation. Tumor volume on EOB $15 \mathrm{~min}$ is the second largest. This might be due to the relatively clear tumor margin secondary to the absorption of Gd-EOB-DTPA by the adjacent normal liver tissues. However, the definite mechanism remains to be confirmed by comparative study on imaging and pathology. Clinically, Portal phase CT can increase the visualization of ICC than non-contrast CT. Previous study has shown that a phase with the best tumor visualization may not correspond to the phase with the largest tumor volume (26). Because perfusion abnormalities can complicate GTV identification and lead to contour heterogeneity. So, we considered that the larger tumor volume on "plain" CT than portal phase CT might 
Table 2 Differences of tumor volume and DSC on different CT and MRI sequences

\begin{tabular}{|c|c|c|}
\hline Parameters & Z & $\mathrm{P}$ \\
\hline \multicolumn{3}{|l|}{ Volume $\left(\mathrm{cm}^{3}\right)$} \\
\hline \multicolumn{3}{|l|}{ Plain } \\
\hline Portal & -1.475 & 0.140 \\
\hline DWI & -2.040 & 0.041 \\
\hline EOB $70 \mathrm{~s}$ & -1.664 & 0.096 \\
\hline EOB $15 \mathrm{~min}$ & -1.036 & 0.300 \\
\hline \multicolumn{3}{|l|}{ Portal } \\
\hline DWI & -2.668 & 0.008 \\
\hline EOB $70 \mathrm{~s}$ & -0.534 & 0.594 \\
\hline EOB $15 \mathrm{~min}$ & -1.726 & 0.084 \\
\hline \multicolumn{3}{|l|}{ DWI } \\
\hline EOB $70 \mathrm{~s}$ & -2.856 & $0.004^{*}$ \\
\hline EOB $15 \mathrm{~min}$ & -1.977 & 0.048 \\
\hline \multicolumn{3}{|l|}{ EOB $70 \mathrm{~s}$} \\
\hline EOB $15 \mathrm{~min}$ & -2.919 & $0.004^{*}$ \\
\hline \multicolumn{3}{|l|}{ DSC } \\
\hline \multicolumn{3}{|l|}{ Plain } \\
\hline Portal & -1.683 & 0.092 \\
\hline DWI & -4.152 & $<0.001^{*}$ \\
\hline EOB $70 \mathrm{~s}$ & -1.705 & 0.088 \\
\hline EOB $15 \mathrm{~min}$ & -1.485 & 0.138 \\
\hline \multicolumn{3}{|l|}{ Portal } \\
\hline DWI & -3.744 & $<0.001^{*}$ \\
\hline EOB $70 \mathrm{~s}$ & -0.457 & 0.648 \\
\hline EOB $15 \mathrm{~min}$ & -0.555 & 0.579 \\
\hline \multicolumn{3}{|l|}{ DWI } \\
\hline EOB $70 \mathrm{~s}$ & -3.026 & $0.002^{*}$ \\
\hline EOB $15 \mathrm{~min}$ & -3.418 & $0.001^{*}$ \\
\hline \multicolumn{3}{|l|}{ EOB $70 \mathrm{~s}$} \\
\hline EOB $15 \mathrm{~min}$ & -0.106 & 0.916 \\
\hline
\end{tabular}

DSC, dice similarity coefficient; Plain, CT scan before the injection of Omnipaque; Portal, CT scan at $70 \mathrm{~s}$ after the injection of Omnipaque; EOB $70 \mathrm{~s}$, mDIXON scan at $70 \mathrm{~s}$ after the injection of Gd-EOB-DTPA; EOB $15 \mathrm{~min}$, mDIXON scan at 15 min after the injection of Gd-EOB-DTPA. *, $\mathrm{P}<0.005$ with nonparametric paired-sample Wilcoxon signed-rank test. be due to the heterogeneous enhancement of ICC on portal phase.

DSC of DWI was significantly larger than that of other CT and MRI sequences. Peripheral hyperintensity and central hypointensity on DWI images with high $b$-value of ICC has been confirmed (25). This might cause the relatively clear boundary of ICC on DWI, which led to better inter-observer repeatability. DSC of EOB $15 \mathrm{~min}$ tended to be larger than that of Plain, Portal and EOB $70 \mathrm{~s}$, however, without significant difference. The potential value of EOB-DTPA in improving the repeatability of GTV delineation on ICC needs further investigation. We also found that DSC was significant positively correlated with tumor volume. This might indicate that larger tumors had better inter-observer agreement on GTV delineation.

DWI and Gd-EOB-DTPA-enhanced MRI showed perfect inter-observer agreements. To our knowledge, ICC on non-contrast CT is usually quite difficult to visualize. Péporté et al. showed that T1-weighted images in the hepatobiliary phase had the highest lesion conspicuity, which was significantly higher than the lesion conspicuity of plain and venous T1-weighted images (8). Xu et al. reported that the detectable rate of ICC on DWI was $100 \%$ (27). In addition, Lewis et al. found that the signal intensity of all ICC lesions increased on DWI with high b-value compared to the normal liver, with diffuse hyperintensity or target appearance (11). Therefore, we considered that the margins might be better seen on DWI and Gd-EOB-DTPAenhanced MRI compared to CT. Compared to CT, MRI allows for better estimation of tumor volume, which can improve the treatment effect of radiotherapy.

This current study has some limitations. First, for a more detailed investigation of the advantage of MRI on GTV delineation, more patients are required. Yet the sample size was comparative to that of a similar investigation of 16 patients and enough for an initial study (28). Second, primary, and recurrent ICCs were not distinguished. Further study is needed to determine whether there is any difference in the GTV delineation between these two groups. Third, though interobserver agreement of GTV is important, whether DWI can more usefully reflect the accurate volume determination is unknown due to the absence of pathological results, which should be further investigated.

\section{Conclusions}

In conclusion, DWI has a significantly higher agreement 
on GTV delineation of ICC. GTV delineations of ICC on Gd-EOB-DTPA-enhanced MRI showed excellent interobserver agreement. Therefore, fusion of CT and MRI images should be considered to improve the accuracy of GTV delineation and reduce radiation-induced liver injury.

\section{Acknowledgments}

Funding: This study has received funding by Key Project and Outstanding Youth supported by Medical Science and Technology Development Foundation Nanjing (YKK15067, QRX11178), Jiangsu Province Key Medical Young Talents, "13th Five-Year" Health Promotion Project of Jiangsu Province (QNRC2016041), and the National Nature Science Foundation of China (81701683). This study was sponsored by Bayer Healthcare Company Ltd.

\section{Footnote}

Conflicts of Interest: All authors have completed the ICMJE uniform disclosure form (available at http:// dx.doi.org/10.21037/qims-19-1093). JH reports grants from Key Project and Outstanding Youth supported by Medical Science and Technology Development Foundation Nanjing, grants from Jiangsu Province Key Medical Young Talents, "13th Five-Year" Health Promotion Project of Jiangsu Province, grants from Bayer Healthcare Company Ltd. FZ reports grants from the National Nature Science Foundation of China, during the conduct of the study. The other authors have no conflicts of interest to declare.

Ethical Statement: The ethical committee of Nanjing Drum Tower Hospital approved this prospective study, and all patients gave written informed consents.

Open Access Statement: This is an Open Access article distributed in accordance with the Creative Commons Attribution-NonCommercial-NoDerivs 4.0 International License (CC BY-NC-ND 4.0), which permits the noncommercial replication and distribution of the article with the strict proviso that no changes or edits are made and the original work is properly cited (including links to both the formal publication through the relevant DOI and the license). See: https://creativecommons.org/licenses/by-nc-nd/4.0/.

\section{References}

1. Chun YS, Javle M. Systemic and Adjuvant Therapies for Intrahepatic Cholangiocarcinoma. Cancer Control 2017;24:1073274817729241.

2. Bridgewater J, Galle PR, Khan SA, Llovet JM, Park JW, Patel T, Pawlik TM, Gores GJ. Guidelines for the diagnosis and management of intrahepatic cholangiocarcinoma. J Hepatol 2014;60:1268-89.

3. Kuhlmann JB, Blum HE. Locoregional therapy for cholangiocarcinoma. Curr Opin Gastroenterol 2013;29:324-8.

4. Chen YX, Zeng ZC, Tang ZY, Fan J, Zhou J, Jiang W, Zeng MS, Tan YS. Determining the role of external beam radiotherapy in unresectable intrahepatic cholangiocarcinoma: a retrospective analysis of 84 patients. BMC Cancer 2010;10:492.

5. Zeng ZC, Tang ZY, Fan J, Zhou J, Qin LX, Ye SL, Sun HC, Wang BL, Li D, Wang JH, Zeng MS, Guo W, Tan YS. Consideration of the role of radiotherapy for unresectable intrahepatic cholangiocarcinoma: a retrospective analysis of 75 patients. Cancer J 2006;12:113-22.

6. El-Bassiouni M, Ciernik IF, Davis JB, El-Attar I, Reiner B, Burger C, Goerres GW, Studer GM. [18FDG] PETCT-based intensity-modulated radiotherapy treatment planning of head and neck cancer. Int J Radiat Oncol Biol Phys 2007;69:286-93.

7. Yang DS, Yoon WS, Lee JA, Lee NK, Lee S, Kim CY, Yim HJ, Lee SH, Chung HH, Cha SH. The effectiveness of gadolinium MRI to improve target delineation for radiotherapy in hepatocellular carcinoma: a comparative study of rigid image registration techniques. Phys Med 2014;30:676-81.

8. Péporté AR, Sommer WH, Nikolaou K, Reiser MF, Zech CJ. Imaging features of intrahepatic cholangiocarcinoma in Gd-EOB-DTPA-enhanced MRI. Eur J Radiol 2013;82:e101-6.

9. Li YT, Cercueil JP, Yuan J, Chen W, Loffroy R, Wáng YX. Liver intravoxel incoherent motion (IVIM) magnetic resonance imaging: a comprehensive review of published data on normal values and applications for fibrosis and tumor evaluation. Quant Imaging Med Surg 2017;7:59-78.

10. Malayeri AA, El Khouli RH, Zaheer A, Jacobs MA, Corona-Villalobos CP, Kamel IR, Macura KJ. Principles and applications of diffusion-weighted imaging in cancer detection, staging, and treatment follow-up. Radiographics 2011;31:1773-91.

11. Lewis S, Besa C, Wagner M, Jhaveri K, Kihira S, Zhu H, Sadoughi N, Fischer S, Srivastava A, Yee E, Mortele K, Babb J, Thung S, Taouli B. Prediction 
of the histopathologic findings of intrahepatic cholangiocarcinoma: qualitative and quantitative assessment of diffusion-weighted imaging. Eur Radiol 2018;28:2047-57.

12. Luo J, Zhou K, Zhang B, Luo N, Bian J. Intravoxel Incoherent Motion Diffusion-Weighted Imaging for Evaluation of the Cell Density and Angiogenesis of Cirrhosis-Related Nodules in an Experimental Rat Model: Comparison and Correlation With Dynamic ContrastEnhanced MRI. J Magn Reson Imaging 2020;51:812-23.

13. Wáng YXJ, Wang X, Wu P, Wang Y, Chen W, Chen H, Li $\mathrm{J}$. Topics on quantitative liver magnetic resonance imaging. Quant Imaging Med Surg 2019;9:1840-90.

14. Nakanuma Y, Sato Y, Harada K, Sasaki M, Xu J, Ikeda H. Pathological classification of intrahepatic cholangiocarcinoma based on a new concept. World J Hepatol 2010;2:419-27.

15. Haradome H, Unno T, Morisaka H, Toda Y, Kwee TC, Kondo H, Sano K, Ichikawa T, Kondo F, Sugitani M, Takayama T. Gadoxetic acid disodium-enhanced MR imaging of cholangiolocellular carcinoma of the liver: imaging characteristics and histopathological correlations. Eur Radiol 2017;27:4461-71.

16. Huppertz A, Haraida S, Kraus A, Zech CJ, Scheidler J, Breuer J, Helmberger TK, Reiser MF. Enhancement of focal liver lesions at gadoxetic acid-enhanced MR imaging: correlation with histopathologic findings and spiral CT-initial observations. Radiology 2005;234:468-78.

17. Tsegmed U, Kimura T, Nakashima T, Nakamura Y, Higaki T, Imano N, Doi Y, Kenjo M, Ozawa S, Murakami Y, Awai K, Nagata Y. Functional image-guided stereotactic body radiation therapy planning for patients with hepatocellular carcinoma. Med Dosim 2017;42:97-103.

18. Lin L, Dou Q, Jin YM, Zhou GQ, Tang YQ, Chen WL, Su BA, Liu F, Tao CJ, Jiang N, Li JY, Tang LL, Xie CM, Huang SM, Ma J, Heng PA, Wee JTS, Chua MLK, Chen H, Sun Y. Deep Learning for Automated Contouring of Primary Tumor Volumes by MRI for Nasopharyngeal Carcinoma. Radiology 2019;291:677-86.

19. van der Veen J, Willems S, Deschuymer S, Robben D, Crijns W, Maes F, Nuyts S. Benefits of deep learning for delineation of organs at risk in head and neck cancer. Radiother Oncol 2019;138:68-74.

20. Fotina I, Lütgendorf-Caucig C, Stock M, Pötter R, Georg D. Critical discussion of evaluation parameters for inter observer variability in target definition for radiation therapy. Strahlenther Onkol 2012;188:160-7.

21. Chung YE, Kim MJ, Park YN, Choi JY, Pyo JY, Kim YC, Cho HJ, Kim KA, Choi SY. Varying appearances of cholangiocarcinoma: radiologic-pathologic correlation. Radiographics 2009;29:683-700.

22. Joo I, Lee JM, Yoon JH. Imaging Diagnosis of Intrahepatic and Perihilar Cholangiocarcinoma: Recent Advances and Challenges. Radiology 2018;288:7-13.

23. Kang Y, Lee JM, Kim SH, Han JK, Choi BI. Intrahepatic mass-forming cholangiocarcinoma: enhancement patterns on gadoxetic acid-enhanced MR images. Radiology 2012;264:751-60.

24. Merkle EM, Zech CJ, Bartolozzi C, Bashir MR, BaSsalamah A, Huppertz A, Lee JM, Ricke J, Sakamoto M, Sirlin CB, Ye SL, Zeng $M$. Consensus report from the 7th International Forum for Liver Magnetic Resonance Imaging. Eur Radiol 2016;26:674-82.

25. Park HJ, Kim YK, Park MJ, Lee WJ. Small intrahepatic mass-forming cholangiocarcinoma: target sign on diffusionweighted imaging for differentiationfrom hepatocellular carcinoma. Abdom Imaging 2013;38:793-801.

26. Niska JR, Keane FK, Wolfgang JA, Hahn PF, Wo JY, Zhu AX, Hong TS. Impact of intravenous contrast enhancement phase on target definition for hepatocellular carcinoma (HCC) and intrahepatic cholangiocarcinoma (IHC): Observations from patients enrolled on a prospective phase 2 trial. Pract Radiat Oncol 2016;6:e9-16.

27. Xu CC, Tang YF, Ruan XZ, Huang QL, Sun L, Li J. The value of Gd-BOPTA- enhanced MRIs and DWI in the diagnosis of intrahepatic mass-forming cholangiocarcinoma. Neoplasma 2017;64:945-53.

28. Helali M, Moreau M, Le Fèvre C, Heimburger C, Bund C, Goichot B, Veillon F, Hubelé F, Charpiot A, Noel G, Imperiale A. 18F-FDOPA PET/CT Combined with MRI for Gross Tumor Volume Delineation in Patients with Skull Base Paraganglioma. Cancers (Basel) 2019;11:54.

Cite this article as: Zhou N, Hu A, Shi Z, Wang X, Zhu Q, Zhou Q, Ma J, Zhao F, Kong W, He J. Inter-observer agreement of computed tomography and magnetic resonance imaging on gross tumor volume delineation of intrahepatic cholangiocarcinoma: an initial study. Quant Imaging Med Surg 2021;11(2):579-585. doi: 10.21037/qims-19-1093 